\title{
Intelligent Adjustment of Printhead Driving Waveform Parameters for 3D Electronic Printing
}

\author{
Na Lin ${ }^{1, a}$, Shikai Jing ${ }^{2, b}$, Hanning Chen $^{3, c}$ and Wenxin $\operatorname{Liu}^{4, d}$ \\ ${ }^{1}$ Beijing Shenzhou Aerospace Software Technology Co. Ltd., Beijing, China \\ ${ }^{2}$ School of Mechanical Engineering, Beijing Institute of Technology, Beijing, China \\ ${ }^{3}$ School of Computer Science and Software, Tianjin Polytechnic University, Tianjin, China \\ ${ }^{4}$ Department of Electrical and Computer Engineering, Lehigh University, Bethlehem, USA. \\ alinn@htrdc.com, bjingshikai@126.com, ${ }^{c}$ perfect_chn@hotmail.com, ${ }^{\mathrm{d}} \mathrm{wliu} @$ lehigh.edu
}

\begin{abstract}
In practical applications of 3D electronic printing, a major challenge is to adjust the printhead for a high print resolution and accuracy. However, an exhausting manual selective process inevitably wastes a lot of time. Therefore, in this paper, we proposed a new intelligent adjustment method, which adopts artificial bee colony algorithm to optimize the printhead driving waveform parameters for getting the desired printhead state. Experimental results show that this method can quickly and accuracy find out the suitable combination of driving waveform parameters to meet the needs of applications.
\end{abstract}

Keywords. Intelligent adjustment, artificial bee colony algorithm, 3D Electronic Printing

\section{Introduction}

In recent years, drop-on-demand (DoD) inkjet printing has achieved a great success in 3D electronic printing [1]. This revolutionary technology can offer a high-resolution, high-speed, solution-thrift and high compatibility way of depositing picoliter droplets with diverse physical and chemical properties on various printing surfaces without contact [2]. Typically, flexible electronics, radio frequency identification (RFID) tags, solar cells, organic light emitting diode (OLED) are the popular applications of inkjet technology in 3D electronic printing engineering [3].

Nowadays, the developments of DoD printing are moving towards higher productivity and quality [4], requiring adjustable small droplet sizes fired at high jetting frequencies. Generally, the print quality delivered by an inkjet printhead depends on the properties of the jetted droplet, i.e., the droplet velocity, the jetting direction, and the droplet volume. However, in order to meet the challenging requirements of printable electronics fabrication, the conductive ink droplet properties have to be tightly controlled for higher inkjet performance [5].

To implement this issue, the theory field and engineering application field follow two different paths for high printing quality. For theory researchers, the analytical or numerical techniques are utilized to model the DoD piezoelectric inkjet printhead. These techniques can offer visualized information about the droplet formation process, such as the acoustic pressure wave traveling inside the channel and ink channel dynamics [6]. Unlike the theory researchers' enthusiasm towards the inkjet mechanism, practice engineering applications need to find an effective means to solve the problem of residual vibrations. A common method is to search an appropriate combination of the parameters of driving waveform for 
the used ink with specific physical properties. Generally, these combinations of the parameters are obtained by exhaustive experiments and a wise guess [7]. However, the search process is extremely unbearable [8]. Especially, when the used ink is replaced, the exhausting search process must be re-established. Therefore, the goal of this work is to shorten the search process with an intelligent method for adjusting the parameters of printhead driving waveform.

In this work, we construct a droplet characteristic optimization system based on image detection method. And then, a canonical swarm intelligent algorithm - artificial bee colony algorithm - is adopted to optimize the combination of printhead driving waveform parameters. This method can quickly and accuracy finds out the suitable combination of driving waveform parameters to meet the needs of applications.

The rest of this paper is organized as follows: In Section 2, a droplet characteristic optimization system based on image detection method is present. Artificial bee colony algorithm (ABC) is introduced in Section 3. Experimental results are described in Section 4. Finally, concluding remarks are collected in Section 5.

\section{Intelligent Adjustment of Printhead Driving Waveform Parameters}

In this experiment, the investigated printhead is KM series IJ Head of Konica and the used ink is nano-silver ink provided by the LEED-PV Corporation, and the according parameters are listed in Table 1.

Table 1. Physical Parameters of Printhead and Ink

\begin{tabular}{|c|c|c|c|}
\hline & Parameter & Unit & Value \\
\hline \multirow{3}{*}{ Printhead } & Nozzle radius, $a_{0}$ & $u m$ & 13.5 \\
\cline { 2 - 4 } & Neck length, $L$ & $u m$ & 45 \\
\hline \multirow{3}{*}{ Ink } & Free electrical capacitance, $\sigma$ & $\mathrm{N} / \mathrm{m}$ & $45^{*} 10^{-3}$ \\
\cline { 2 - 4 } & Viscosity, $u$ & $\mathrm{Kg} /\left(\mathrm{m}^{*} \mathrm{~s}\right)$ & 15 \\
\cline { 2 - 4 } & Density, $\rho_{0}$ & $\mathrm{Kg} / \mathrm{m}^{3}$ & $1.05^{*} 10^{3}$ \\
\cline { 2 - 4 } & Sonic speed, $C_{0}$ & $\mathrm{~m} / \mathrm{s}$ & 1400 \\
\hline
\end{tabular}

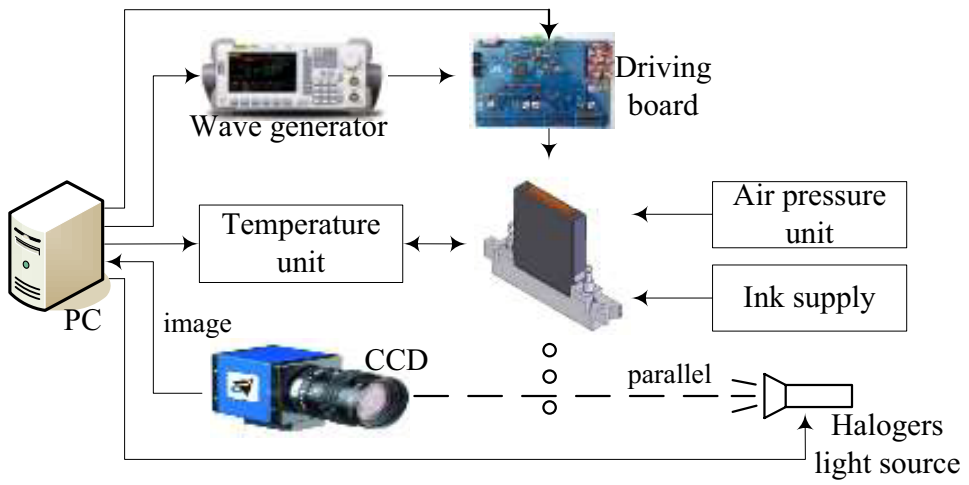

Fig. 1. Schematic of experimental setup.

A schematic overview of image detection system to observe thedroplet formation process is shown in Figure 1. The visualization system consists of a cold source of halogens and a CCD camera. The volume andvelocity of droplet and the profile of meniscus are measured through the images captured by CCD. The high-frequency driving waveform is programmed by arbitrary wave generator. In orderto repeatedly generate uniform droplets, ink supply unit connects air pressure unit, which offers suitable negative force to prevent the ink leaking from the printhead.

Here, we apply the $\mathrm{ABC}$ algorithm to search theappropriate combinations of the driving waveform parameters. A schematic diagram of the approach isillustrated in Figure 2. 


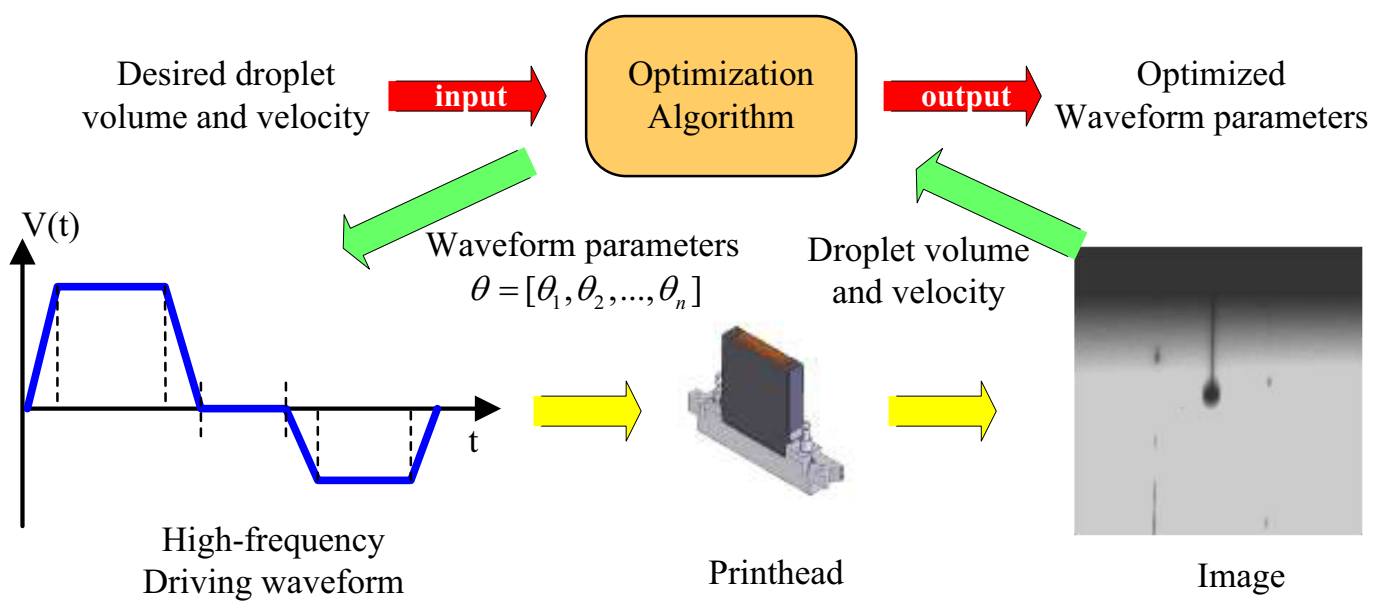

Fig.2. A schematic diagram of optimization framework for the appropriate combination of waveform parameters based on image analysis

The driving waveform input the PZT printhead is parameterized in a vector with several dimensions. The printhead works under the corresponding driving waveform and the pressure is generated in the ink channel. The pressure drives the ink out of the nozzle and the liquid filament is formed. The image detection system capture the image of droplet formation process, and the droplet volume and velocity are calculated. Through optimizing procedure with $\mathrm{ABC}$ algorithm, the optimal pulse parameters are obtained by minimizing the error betweenthe desired droplet volume (or velocity) and measured droplet volume (or velocity).

\section{Artificial Bee Colony algorithm}

The artificial bee colony (ABC) algorithm was firstly presented by Karaboga in 2005 [9], motivated by the intelligent foraging behavior of honeybees. The entire bee-colony is classified into three types of bees: employed bees, onlooker bees and scout bees. The employed bees explore the specificnectar (food) sources; meanwhile pass the food information to onlooker bees. Each food source corresponds to only one employed bee, which means the population size of employed bees is equal to the number of food sources. The onlooker bees are responsible forselecting good food sources, those with higher quality having a bigger chance to be chosen by onlookers. And then they further exploit the food near their selected food sources. If a food source found by the employed bee is exhausted, it will be replaced with a new one by a newly produced scout. The fundamental mathematic representations are listed as follows.

Step.1 Initialization phase

In the initialization phase, the $\mathrm{ABC}$ algorithm generates a randomly distributed initial food source positions of $S N$ solutions, where $S N$ denotes the size of employed bees or onlooker bees. Each solution $x_{i}(\mathrm{i}=1,2, \ldots, S N)$ is a $D$-dimensional vector. Here, $D$ is the number of optimization parameters. And then evaluate each nectar amount fitness $i_{i}$. In $\mathrm{ABC}$ model, nectar amount is the solution value of benchmark function or real-world problem.

Step. 2 Employed bees' phase

In the employed bees' phase, the neighbor food source (candidate solution) can be generated from the old food source of each employed bee in its memory using the following expression:

$$
v_{i, j}=x_{i, j}+\varphi\left(x_{i, j}-x_{k, j}\right)
$$


where $k$ is a randomly selected food source and must be different from $i ; j$ is a randomly chosen index; $\varphi$ is a random number in range $[-1,1]$.

Step.3 Onlooker bees' phase

In the onlooker bees' phase, one onlooker bee selects a food source depending on the probability value associated with that food source, $P_{i}$ can be calculated as follows:

$$
P_{i}=\frac{\text { fitness }_{i}}{\sum_{j=1}^{S N} \text { fitness }_{j}}
$$

where fitness $i$ is the fitness value of $j$ th solution.

Table 2. Pseudocode of the canonical $\mathrm{ABC}$ algorithm

The canonical $\mathrm{ABC}$ algorithm

1: Initialization.

Initialize the food sources and evaluate the nectar amount (fitness) of food sources;

Send the employed bees to the current food source;

2:teration $=0$;

3: Do while(the termination conditions are not met)

4: $\quad / * E m p l o y e d ~ B e e s '$ Phase*/

for (each employed bee)

find a new food source in its neighborhood following the Eq. (2);

Evaluate the fitnessof the new food source, Apply greedy selection;

end for

5: Calculate the probability P for each food source;

6: $\quad / *$ Onlooker Bees'Phase*/

for (each onlooker bee)

Send onlooker beesto food sources depending on P;

finda new food source in its neighborhood following the Eq. (2);

Evaluate the fitnessof the new food source, Apply greedy selection;

end for

7: $\quad$ /*Scout Bees' Phase*/

if (any employed bee becomes scout bee)

Send the scout bee to a randomlyproduced food source;

end if

8: Memorize the best solution achieved so far

Iteration $=$ Iteration +1 ;

end while

9: Output the best solution achieved

Step.4 Scout bees' phase

In the scout bee phase, if a food source cannot be improved further through a predetermined number of cycles, then that food source is assumed tobe abandoned. The value of predetermined number of cycles is an important control parameter of $\mathrm{ABC}$, which is called limit for abandonment. Then the food source abandoned by the bees is replaced with a new food source by the scouts. A new food source will be produced randomly in the search space usingtheequationbelow:

$$
x_{i, j}=x_{\min , j}+\operatorname{rand}[0,1]\left(x_{\max , j}-x_{\min , j}\right)
$$

where $x^{j}{ }_{\text {min }}$ and $x^{j}{ }_{\text {max }}$ are lower and upper bounds of parameter $j$, respectively. 
The employed, onlooker and scout bees' phase will recycle until the termination condition is met. The pseudocode of the $\mathrm{ABC}$ algorithm is listed in Table 2.

\section{Experimental results}

In order to reflect the error between the optimized droplet characteristics and the desired droplet characteristics, the fitness function for calculating such error should be set first. The fitness function is defined as:

$$
F=\min \left[w(1)\left(V-V_{\text {target }}\right)^{2}-w(2)\left(S-S_{\text {target }}\right)^{2}\right]
$$

where $V_{\text {target }}$ is the desired droplet velocity, $V$ is the optimized droplet velocity, $S_{\text {target }}$ is the desired droplet volume, $S$ is the optimized droplet volume, and $w(1)$ and $w(2)$ are the user-defined weights.

The desired droplet characteristics include droplet volumesmaller than $1.2 \times 10^{-5} \mathrm{~L}$ and the droplet velocity largerthan $5 \mathrm{~m} / \mathrm{s}$. The weight is set as $w=[0.2,0.8]$.

In order to show the optimize accuracy, the convergence curve of artificial bee colony algorithm for optimizing the desired droplet characteristics is shown in Figure 3.

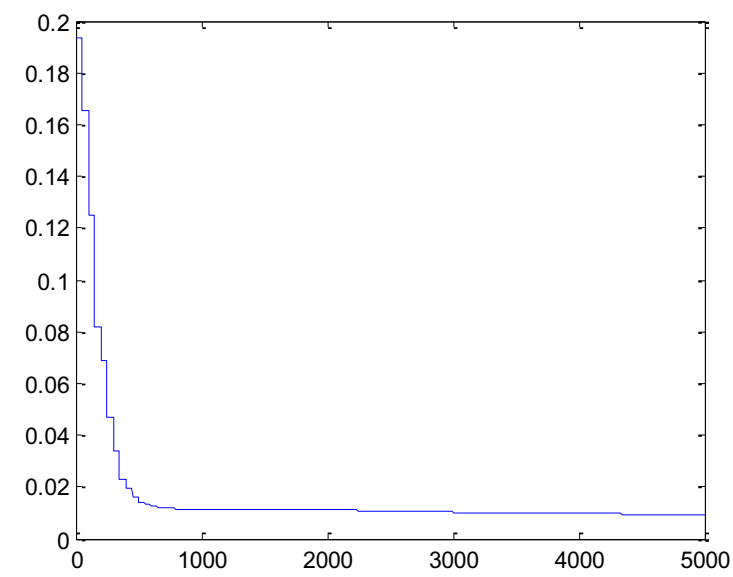

Fig.3. Convergence curve of optimization the desired droplet characteristics by artificial bee colony algorithm.

From Figure 3, it is obvious that the droplet characteristics (droplet velocity and droplet volume) gradually meet the desired values ( $V_{\text {target }}$ and $\left.S_{\text {target }}\right)$. The final optimized results is that the droplet velocity is $5.06 \mathrm{~m} / \mathrm{s}$, the droplet volume is $11.83 \mathrm{pL}$ and the fitness value is 0.0087 . The optimized results can satisfied the practical needs of $3 \mathrm{D}$ electronic printing.

\section{Conclusion}

In this paper, we proposed a new intelligent method to optimize the parameters of printhead driving waveform for getting the desired droplet characteristics. The optimization algorithm is a canonical artificial bee colony algorithm. Experimental results shown that the proposed adjustment method can quickly and accuracy find out the suitable combination of printhead driving waveform parameters. The method can effectively replace the traditional manual search process based on exhaustive experiments and a wise guess.

\section{Acknowledgements}

This research is partially supported by National Natural Science Foundation of China (Grand Nos. 51575158, and 61305028), and a 
self-planned task of CAS Key Laboratory of Networked Control System (Grand No. WLHKZ2015001).

\section{References}

1. M. Singh, H.M. Haverinen, Inkjet printing: Process and its applications. Advanced Materials, Vol.22 (2010), p. 673-685.

2. E. Macdonald, R. Salas, D. Espalin, M. Perez, E. Aguilera, D. Muse, R.B. Wicker, 3D printing for the rapid prototyping ofstructural electronics. IEEE Access, Vol. 2 (2014), p. 234-242.

3. H. Jaehyung, W. Alan, K. Antoine. Energetics of metal-organic interfaces: New experiments and assessment of the field, Materials Science and Engineering, Vol. 64(2009), p. 1-31.

4. J.C. Lioua, F. Tsenga. Multi-dimensional data registration CMOS/MEMS integrated inkjet printhead. Journal of Microelectro mechanical Systems, Vol.19, no.4 (2011), p.961-972.

5. J. Miettinen, K.Kaija, M. Mantysalo, P. Mansik kamaki, Molded substrates for inkjet printed modules. IEEE Transactions onComponents and Packaging Technologies, Vol.32 (2009), p. 293-301.

6. L. Sang, Y. Hong, F. Wang. Investigatin of viscosity effect ondroplet formation in T-shaped micro-channels by numerical andanalytical methods. Microfluidics and Nanofluidics, Vol.6 (2009), p. 6621-6635.

7. N.Reis, C. Ainsley, B. Derbyb, Ink-jet delivery of particlesuspensions by piezoelectric droplet ejectors. Journal of Applied Physics, Vol. 97 (2005), p. 094903-1-6.

8. K. Kwon, Waveform design methods for piezo inkjet dispensers based on measured meniscus motion. Journal of Microelectro mechanical Systems, Vol. 18, no.5 (2009), p. 1118-1125.

9. D. Karaboga, B. Basturk, On the performance of Artificial Bee Colony (ABC), Applied Soft Computing, Vol. 8, no.1 (2008), p. 687-697. 